\title{
Downregulation of amine oxidase copper containing 1 inhibits tumor progression by suppressing IL-6/JAK/STAT3 pathway activation in hepatocellular carcinoma
}

\author{
QIAN DING $^{1^{*}}$, DONGDONG LIN ${ }^{2 *}$, YAJING ZHOU $^{3}$, FENG LI $^{1}$, JIANMING LAI $^{4}$, \\ JIANPING DUAN $^{1}$, JING CHEN $^{5}$ and CAIHUA JIANG ${ }^{6}$
}

${ }^{1}$ Department of Infectious Disease; ${ }^{2}$ Blood Purification Center; ${ }^{3}$ Department of Physical Therapy, Qingdao No. 6 People's Hospital, Qingdao, Shandong 266033; ${ }^{4}$ School of Clinical Medicine, QingDao University Medical College,

Qingdao, Shandong 266071; ${ }^{5}$ Department of Eight Areas of Liver Disease; ${ }^{6}$ Outpatient Department, Qingdao No. 6 People's Hospital, Qingdao, Shandong 266033, P.R. China

Received January 19, 2021; Accepted July 30, 2021

DOI: $10.3892 / 01.2021 .13118$

\begin{abstract}
Amine oxidase copper containing 1 (AOC1) is a copper-containing amine oxidase that catalyzes the deamination of polyamines. AOC1 functions as an oncogene in human gastric cancer. There is little information available regarding the function of $\mathrm{AOC} 1$ in hepatocellular carcinoma (HCC). In the present study, reverse transcription-quantitative PCR was used to detect the expression levels of AOC1 in HCC tissues, and the role of $\mathrm{AOC} 1$ in $\mathrm{HCC}$ progression was determined using western blot, Cell Counting Kit 8, clone formation, wound-healing and Transwell assays. An AOC1 survival curve was generated with data downloaded from The Cancer Genome Atlas, and Gene Set Enrichment Analysis was performed to investigate the potential biological mechanisms of $\mathrm{AOC} 1$ in HCC. AOC1 was found to be upregulated in HCC tissues, which was associated with a poor prognosis. Furthermore, AOC1-knockdown inhibited HCC cell proliferation, migration and invasiveness, suppressed IL-6 expression, as well as decreasing JAK2 and STAT3 phosphorylation. Ultimately, the results of the present study illustrate that $\mathrm{AOC} 1$ promoted
\end{abstract}

Correspondence to: Dr Jing Chen, Department of Eight Areas of Liver Disease, Qingdao No. 6 People's Hospital, 9 Fushun Road, Shibei, Qingdao, Shandong 266033, P.R. China

E-mail: jingcn996@163.com

Dr Caihua Jiang, Outpatient Department, Qingdao No. 6 People's Hospital, 9 Fushun Road, Shibei, Qingdao, Shandong 266033, P.R. China

E-mail: 361238247@qq.com

*Contributed equally

Key words: amine oxidase copper containing 1, proliferation, invasion, migration, IL-6/JAK/STAT3, epithelial-mesenchymal transition the proliferation, migration and invasiveness of HCC cells by regulating the IL-6/JAK/STAT3 pathway.

\section{Introduction}

Liver cancer is the fourth leading cause of cancer-related mortality worldwide, accounting for 782,000 deaths in 2018 (1). Hepatocellular carcinoma (HCC) accounts for $\sim 75-85 \%$ of all primary liver cancers (2). Various risk factors are associated with HCC, including hepatitis virus infection, excessive consumption of alcohol, and consumption of food stuffs contaminated with aflatoxin B1 (3). Treatment options for patients with HCC include surgical resection, liver transplantation, locally destructive therapies and systemic chemotherapy (4). However, HCC is often diagnosed at a later stage when surgery is no longer on option (5), and patients may experience recurrent pain following liver resection (6). Therefore, it is essential to investigate the pathogenesis of HCC to facilitate further informed therapeutic interventions.

Since the oxidation products of biogenic amines are believed to be carcinogenic, amine oxidases have been suggested to participate in cancer progression (7). The copper/TPQ-containing amine oxidases include amine oxidase copper-containing 1 (AOC1), retina-specific amine oxidase (AOC2), vascular adhesion protein-1 (AOC3) and serum amine oxidase (AOC4) (8). AOC3 expression is upregulated in patients with breast cancer (luminal B and HER-2), where it is associated with lymph node metastasis (9). Furthermore, AOC3-targeted inhibitors have been developed to treat inflammatory diseases and cancer (10), and $\mathrm{AOC} 1$ promotes gastric cancer progression in humans (11). However, the role of $\mathrm{AOC1}$ in $\mathrm{HCC}$ progression remains unclear.

In the present study, gene set enrichment analysis (GSEA) and functional enrichment analysis were performed. Mitochondrial reactive oxygen species (ROS) production, which inhibits the JAK2/STAT3 signaling pathway, serves a role in the anticancer effect of drugs $(12,13)$. Monoamine oxidase A (MAO-A) activates the IL-6/STAT3 pathway, which promotes the proliferation of tumor cells (14). Lee et al (15) 
reported that $\mathrm{MAO}-\mathrm{A}$ was downregulated in nasopharyngeal carcinoma tissues and EBV-infected nasopharyngeal carcinoma cells through the IL-6/IL-6R/STAT3 pathway, and that this may be attributed to EBV infection in these cells. As such, the IL-6/JAK/STAT3 pathway, which is downstream of AOC1, was selected for investigation.

The aim of the present study was to investigate the effects of $\mathrm{AOC} 1$ on $\mathrm{HCC}$ cell proliferation, migration and invasiveness. Reverse transcription-quantitative (RT-q) PCR was used to detect the expression levels of AOC1 in HCC tissues, and the role of $\mathrm{AOC} 1$ in $\mathrm{HCC}$ progression was determined using western blotting, Cell Counting Kit 8 (CCK-8), clone formation, wound-healing and Transwell assays. An AOC1 overall survival (OS) curve was generated with data downloaded from The Cancer Genome Atlas (TCGA), and GSEA was performed to investigate the potential biological mechanisms of $\mathrm{AOC} 1$ in $\mathrm{HCC}$.

\section{Materials and methods}

Bioinformatic analysis. An AOC1 survival curve was generated with the $\mathrm{R}$ package 'survival' using data downloaded from TCGA database (16). Tumor tissues sample data $(n=374)$ were downloaded from LIHC-TCGA database (https://portal. gdc.cancer.gov/). A total of 140 samples with $\mathrm{OS} \leq 30$ days were excluded, as these patients may succumb to nonneoplastic factors. As such, 234 samples were divided into two groups AOC1 high expression $(n=117)$ and AOC1 low expression $(n=117)$ groups; cut-off, 5.16). To investigate the potential biological mechanisms of AOC1 in HCC, GSEA was performed using the $\mathrm{R}$ package 'clusterProfiler'; data form 50 normal and 374 tumor patients were downloaded from LIHC-TCGA (https://portal.gdc.cancer.gov/). The annotated gene set file (h.all.v7.0.entrez.gmt, http://www.gsea-msigdb. org/gsea/downloads.jsp) was used as the reference for analysis.

Tissue samples. Tumor tissues and adjacent-normal tissues were collected from 85 patients who underwent surgery at Qingdao No. 6 People's Hospital (Qingdao, China) between December 2016 and December 2018. The inclusion criteria for patients stipulated that examination results meet the diagnostic criteria for HCC. Patients must have been initially diagnosed with HCC, and not received any treatment before surgery. Patients who had received allogeneic blood transfusion or cellular immunotherapy within 1 year of study commencement were excluded. Adjacent-normal tissues were collected $\geq 2 \mathrm{~cm}$ from the tumor lesions. The clinical characteristics of the patients were reviewed by two independent pathologists according to the World Health Organization grading system (17). All tissues were fragmented into $0.1 \mathrm{~cm}^{3}$ pieces and stored at $-80^{\circ} \mathrm{C}$ for long-term preservation. The present study was approved by the Human Research Ethics Committee of Qingdao No. 6 People's Hospital (approval no. 201863), and written informed consent was obtained from all patients.

Cell culture. Human HCC cells (Huh-7 and Hep3B2.1-7) were selected as previously described (18), and obtained from the Chinese Academy of Sciences (Shanghai, China). The cells were maintained in RPMI 1640 (Gibco; Thermo Fisher Scientific, Inc.) containing $10 \%$ fetal bovine serum
(FBS), $100 \mathrm{U} / \mathrm{ml}$ penicillin and $100 \mathrm{mg} / \mathrm{ml}$ streptomycin (all sourced from Hyclone; Cytiva), at $37^{\circ} \mathrm{C}$ in an atmosphere with $5 \% \mathrm{CO}_{2}$. Cells were pretreated with $100 \mathrm{ng} / \mathrm{ml}$ human IL-6 (Sigma-Aldrich; Merck KGaA) for $48 \mathrm{~h}$ prior to subsequent experimentation.

Transfection. Huh-7 and Hep3B2.1-7 cells were transfected with $20 \mathrm{nM}$ small interfering (si)RNAs targeting AOC1, the corresponding negative control (siNC), pcDNA3.1-AOC1 or the empty pcDNA3.1 vector (Guangzhou RiboBio Co., Ltd.), using Lipofectamine ${ }^{\circledR} 2000$ (Invitrogen; Thermo Fisher Scientific, Inc.) at $37^{\circ} \mathrm{C}$ for $6 \mathrm{~h}$. Huh-7 and Hep3B2.1-7 cells were harvested $48 \mathrm{~h}$ after transfection. The siRNA sequences are listed in Table I.

$R T$ - $q$ PCR. Total RNA was extracted from HCC tissue samples and cells using TRIzol ${ }^{\circledR}$ reagent (Invitrogen; Thermo Fisher Scientific, Inc.). Then, $1 \mu \mathrm{g}$ RNA was reverse-transcribed using the iScript cDNA Synthesis kit (Bio-Rad Laboratories, Inc.). Gene expression level was determined using the SYBR Premix Ex Taq kit (Takara Bio, Inc.) with an ABI 7500 instrument (Applied Biosystems; Thermo Fisher Scientific, Inc.). The qPCR thermocycling conditions were as follows: $95^{\circ} \mathrm{C}$ for $10 \mathrm{~min} ; 40$ cycles of $95^{\circ} \mathrm{C}$ for $10 \mathrm{sec}, 60^{\circ} \mathrm{C}$ for $30 \mathrm{sec}$, and $75^{\circ} \mathrm{C}$ for $30 \mathrm{sec}$. Relative gene expression was calculated using the $2^{-\Delta \Delta \mathrm{Cq}}$ method (19), and the qPCR primer sequences are listed in Table II.

CCK-8 assay. The CCK-8 kit (Dojindo Molecular Technologies, Inc.) was used to evaluate cell viability. Transfected Huh-7 and Hep3B2.1-7 cells $\left(2 \times 10^{4} / \mathrm{ml}\right)$ were added to 96 -well plates. Then, $10 \mu \mathrm{l}$ of CCK-8 solution was added into each well, followed by incubation for $2 \mathrm{~h}$ at $37^{\circ} \mathrm{C}$. Absorbance was recorded at $450 \mathrm{~nm}$ using a microplate reader (Molecular Devices, LLC).

Colony formation assay. Transfected Huh-7 and Hep3B2.1-7 cells (200 cells/well) were seeded into 12-well plates. After 2 weeks at $37^{\circ} \mathrm{C}$, colonies were fixed with $4 \%$ paraformaldehyde at $25^{\circ} \mathrm{C}$ for $15 \mathrm{~min}$, and then stained with $0.1 \%$ crystal violet (at $25^{\circ} \mathrm{C}$ for $15 \mathrm{~min}$. Finally, the number of colonies (cell clusters containing $\geq 5$ cells) were counted manually using a microscope (DMI3000 B; Leica Microsystems, Inc.; magnification, $\mathrm{x} 100)$.

Wound-healing analysis. Transfected Huh-7 and Hep3B2.1-7 cells $\left(1 \times 10^{5}\right)$ were seeded into a 6-well plate, cultured for $24 \mathrm{~h}$, and then treated with $10 \mu \mathrm{g} / \mathrm{ml}$ Mitomycin C (Thermo Fisher Scientific, Inc.) for $2 \mathrm{~h}$ to halt proliferation. A wound was created through the monolayer using a $100-\mu 1$ pipette tip, and the cells were cultured in serum-free medium. Wound-closure images were captured under a microscope (DMI3000 B; Leica Microsystems, Inc.; magnification, $\mathrm{x} 100)$ at 0 and $24 \mathrm{~h}$. The wound healing rate was calculated as follows: ( $0 \mathrm{~h}$ width of scratch-24 $\mathrm{h}$ width of scratch $) / 0 \mathrm{~h}$ width of scratch $\times 100 \%$.

Transwell assays. Transwell chambers (Corning, Inc.) were precoated with Matrigel at $37^{\circ} \mathrm{C}$ for $60 \mathrm{~min}$. Transfected Huh-7 and Hep3B2.1-7 cells $\left(4 \times 10^{4} / \mathrm{ml}\right)$ were added to the 
Table I. SiRNA sequences.

\begin{tabular}{lc}
\hline Name & \multicolumn{1}{c}{ Sequence } \\
\hline siAOC1-1 & 5'-GAATGGTATAAGCAAGGAGTA-3' \\
siAOC1-2 & 5'-GGTTGAGAAGTAGGTCATTGA-3' \\
siAOC1-3 & 5'-GACTACATGGCAGGCAATATA-3' \\
siNC & 5'-GAATAACATGGATGGGATAGA-3'
\end{tabular}

Table II. Quantitative PCR primer sequences.

\begin{tabular}{ll}
\hline Primer names & \multicolumn{1}{c}{ Sequence } \\
\hline AOC1 forward & 5'-TGTCCACGCAACCTTCTACA-3' \\
AOC1 reverse & 5'-ACTGGGTCTGCTCAAGTGTG-3' \\
GAPDH forward & 5'-TTTGGTCGTATTGGGCGCCTGG \\
& TCA-3' \\
GAPDH reverse & 5'-TTGTGCTCTTGCTGGGGCTGGT \\
& GGT-3' \\
\hline
\end{tabular}

AOC1, amine oxidase copper containing 1.

upper chamber and incubated with serum-free medium, while medium containing $10 \%$ FBS was added to the lower chamber. After $24 \mathrm{~h}$ of incubation at $37^{\circ} \mathrm{C}$, the invaded cells were stained using $0.1 \%$ crystal violet at $25^{\circ} \mathrm{C}$ for $15 \mathrm{~min}$, and counted using a microscope (DMI3000 B; Leica Microsystems, Inc.; magnification, $\mathrm{x} 100)$.

Western blotting. The total protein was extracted from tissues and cells using RIPA buffer (Beyotime Institute of Biotechnology). Protein concentration was determined using a BCA kit (Beyotime Institute of Biotechnology) Then, total protein $(50 \mu \mathrm{g})$ was separated using $10 \%$ SDS-PAGE and transferred to polyvinylidene difluoride membranes (MilliporeSigma). The membranes were blocked with $5 \%$ fat-free milk at $25^{\circ} \mathrm{C}$ for $1 \mathrm{~h}$, and then incubated with primary antibodies overnight at $4^{\circ} \mathrm{C}$, including AOC1 (cat. no. ab231558; Abcam), E-cadherin [cat. no. \#3195; Cell Signaling Technology (CST)], N-cadherin (cat. no. \#13116; CST), vimentin (cat. no. \#5741; CST), JAK2 (cat. no. \#3230; CST), p-JAK2 (Tyr1007/1008; cat. no. \#3776; CST), STAT3 (cat. no. \#12640; CST) and p-STAT3 (Tyr705; cat. no. \#9145; CST) (all 1:1,000). The membranes were then incubated with an HRP-conjugated secondary antibody (1:1,000; cat. no. ZB-5301, Zhongshan Goldenbridge Biotechnology, Inc.) for $1 \mathrm{~h}$ at room temperature. Finally, the blots were visualized using an enhanced chemiluminescence reagent (Sigma-Aldrich; Merck KGaA) and a chemiluminescence system (Bio-Rad Laboratories, Inc.). Protein expression was quantified using Image-Pro ${ }^{\circledR}$ Plus software (version 6.0; Media Cybernetics, Inc.).

Detection of reactive oxygen species (ROS). Transfected Huh-7 and Hep3B2.1-7 cells $\left(1 \times 10^{5}\right)$ cultured in 6-well plates were incubated with $10 \mu \mathrm{M}$ 2',7'-dichlorofluorescein diacetate
(Beyotime Institute of Biotechnology) for $30 \mathrm{~min}$ at room temperature in the dark. After washing, the cells were analyzed using a flow cytometer (BD Accuri C6 Plus; BD Biosciences). The FlowJo software (version 10.7.1; FlowJo, LLC) was used for data analysis.

ELISA. Concentrations of IL-6 in the cell supernatants were detected using an IL-6 ELISA kit (cat. no. PI330, Beyotime Institute of Biotechnology) per the manufacturer's protocol. Absorbance was measured at $450 \mathrm{~nm}$ using a microplate reader (Molecular Devices, LLC).

Statistical analysis. All experiments were conducted at least three times, and the data are presented as the mean \pm standard error of the mean. Statistical analysis was conducted using GraphPad Prism 7 (GraphPad Software, Inc.). The association between clinical characteristics and $\mathrm{AOC} 1$ expression was evaluated using Fisher's exact test, and survival analysis was performed using the Kaplan-Meier method; the log-rank test was used to determine statistical significance between two groups. Two-tailed paired Student's t-test was used to determine the statistical differences in AOC1 expression between HCC and adjacent-normal tissues. One-way variance analysis and Tukey's post hoc test were used to compare the differences among multiple groups. $\mathrm{P}<0.05$ was considered to indicate a statistically significant difference.

\section{Results}

AOCl is upregulated in HCC tissues. To examine the role of AOC1 in HCC, SNHG4 expression was measured in $\mathrm{HCC}$ tissues. AOC1 expression was upregulated in HCC tissue samples compared with adjacent-normal tissues (Fig. 1A). According to TCGA data, the results of Kaplan-Meier curve analysis indicated that patients with high AOC1 expression levels had a poorer overall survival rate than those with low expression (Fig. 1B). Furthermore, the expression of AOC1 was associated with poor tumor differentiation, advanced clinical stage and lymph node metastasis, while no notable difference was found in relation to sex, age, hepatitis B virus infection, liver cirrhosis and tumor size (Table III). Therefore, the data suggested that AOC1 may promote HCC development.

AOC1-knockdown inhibits HCC cell proliferation. To understand the effect of AOC1 in HCC, cell viability and colony formation were evaluated when $\mathrm{AOC} 1$ expression was downregulated or upregulated in Huh-7 and Hep3B2.1-7 cells. Knockdown and overexpression of AOC1 in Huh-7 and Hep3B2.1-7 cells was confirmed using RT-qPCR and western blotting (Fig. 2A and B). Huh-7 and Hep3B2.1-7 cell proliferation decreased in response to transfection with si-AOC1, but increased in response to transfection with pcDNA3.1-AOC1 (Fig. 2C). The colony formation assay revealed suppressed proliferative ability in the Huh-7 and Hep3B2.1-7 cells treated with si-AOC1-1 and si-AOC1-2, and increased ability following treatment with pcDNA3.1-AOC1 (Fig. 2D). Therefore, downregulation of $\mathrm{AOC} 1$ inhibited $\mathrm{HCC}$ cell proliferation.

AOC1-knockdown inhibits HCC cell migration and invasiveness. The migration and invasiveness of Huh-7 and Hep3B2.1-7 cells 
Table III. Association between AOC1 expression and clinical characteristics of patients with hepatocellular carcinoma.

\section{AOC1 expression}

Clinical characteristic

Patients $(n=85)$

High $(n=42)$

Low (n=43)

P-value

Sex

Male
Female

Age (years)

$\geq 50$

$<50$

Hepatitis B virus infection

Positive

Negative

Liver cirrhosis

Present

Absent

$\alpha$-fetoprotein

$\geq 20 \mathrm{ng} / \mathrm{ml}$

$<20 \mathrm{ng} / \mathrm{ml}$

Tumor size

$\geq 3 \mathrm{~cm}$

$<3 \mathrm{~cm}$

Degree of tumor differentiation

Poorly + moderately

Well

TNM staging

I-II

III-IV

Lymph node metastasis

Yes

No
51

34

49

36

80

5

78

7

57

28

42

43

62

23

53

32

24

24
18

18

24

18

41

1

37

5

30

12

22

20

37

5

21

21

28

57
0.6610

27

16

25

18

39

4

41

2

27

16

0.6665

20

23

0.0030

25

18

0.0259

32

11

AOC1, amine oxidase copper containing 1 .
A

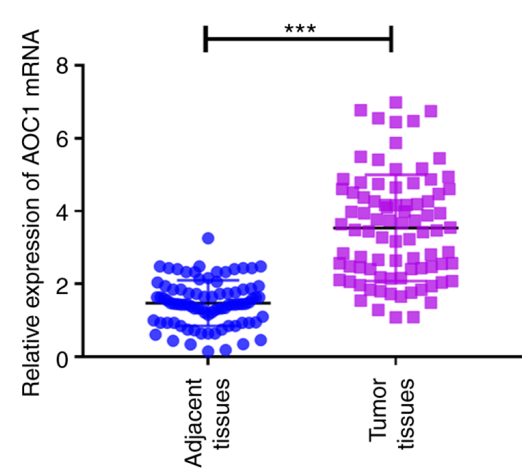

B

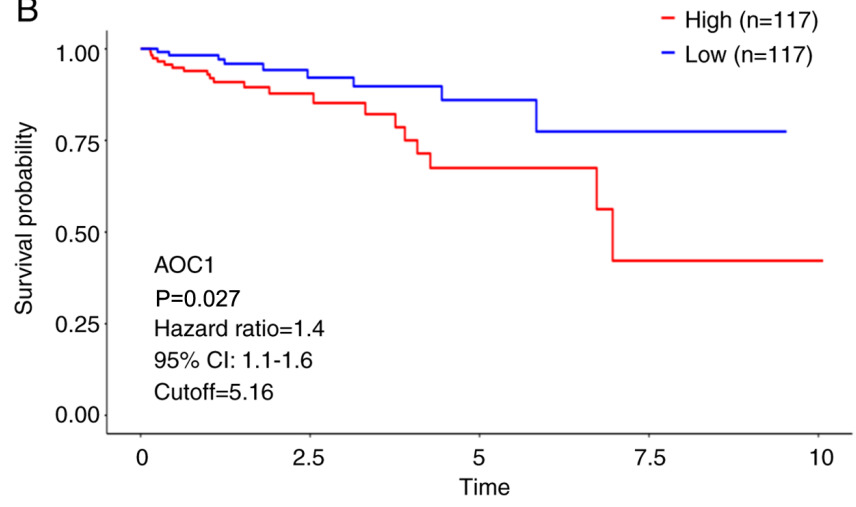

Figure 1. AOC1 expression levels in HCC tissues. (A) AOC1 mRNA expression in 85 HCC and adjacent-normal liver tissues. (B) Overall survival of 234 patients with HCC based on data from The Cancer Genome Atlas. ${ }^{* * * *} \mathrm{P}<0.001$. AOC1, amine oxidase copper containing 1 ; HCC, hepatocellular carcinoma.

were investigated, and the results revealed that knockdown of AOC1 significantly suppressed the migration and invasion abilities of Huh-7 and Hep3B2.1-7 cells, while AOC1 overexpression significantly promoted these characteristics (Fig. 3A and B). 
A

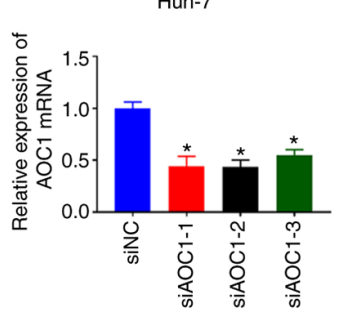

Hep3B2.1-7

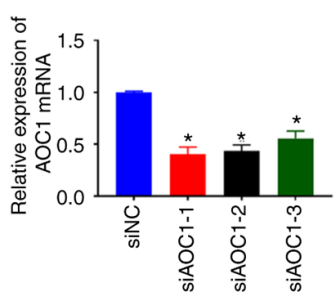

Huh-7

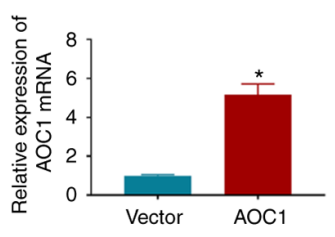

Hep3B2.1-7

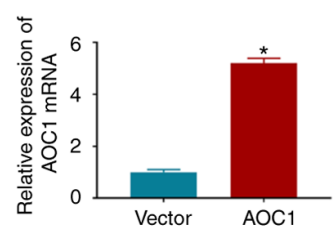

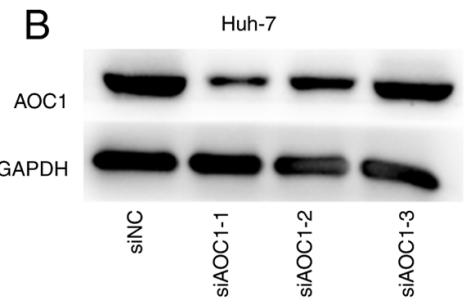

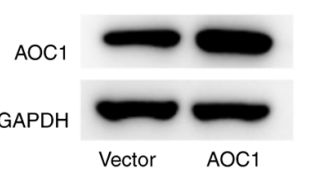

C

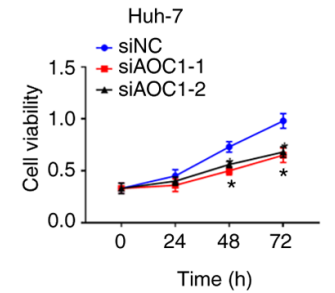

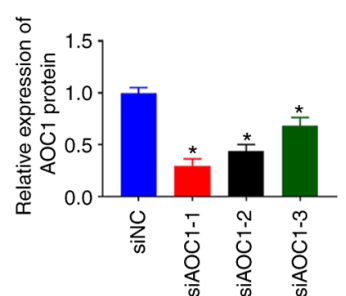

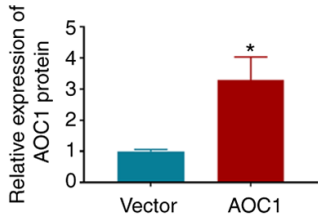

Hep3B2.1-7

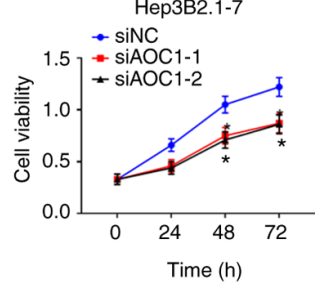

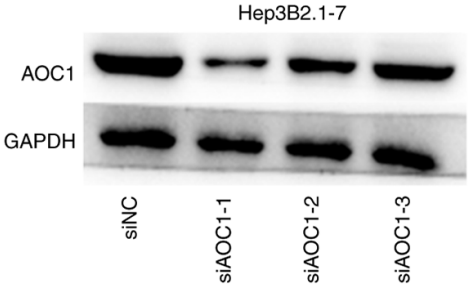
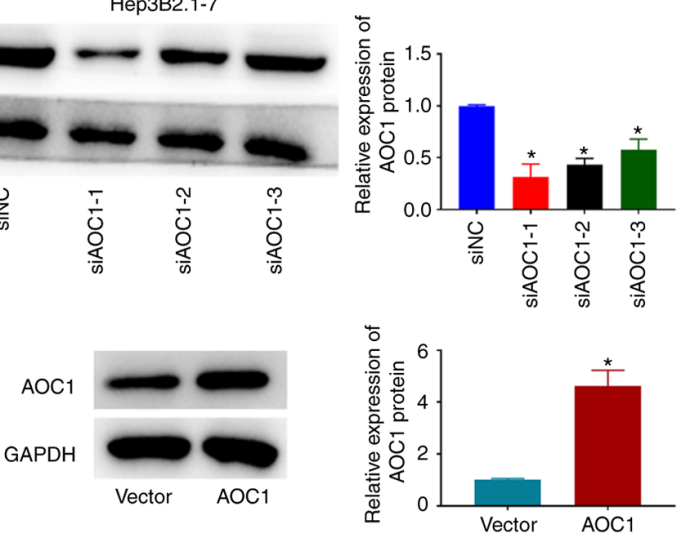

Huh-7

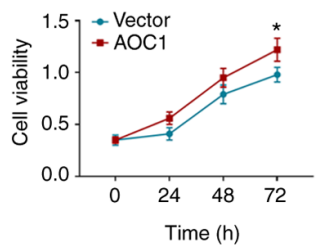

Нер3В2.1-7

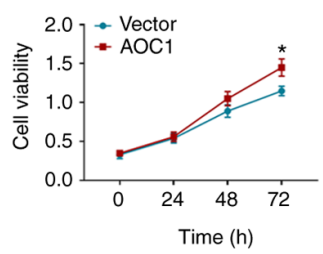

Huh-7
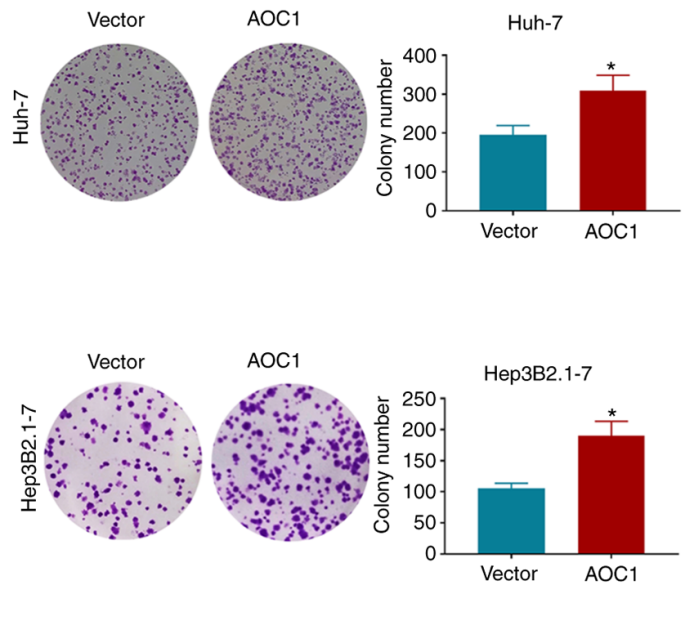
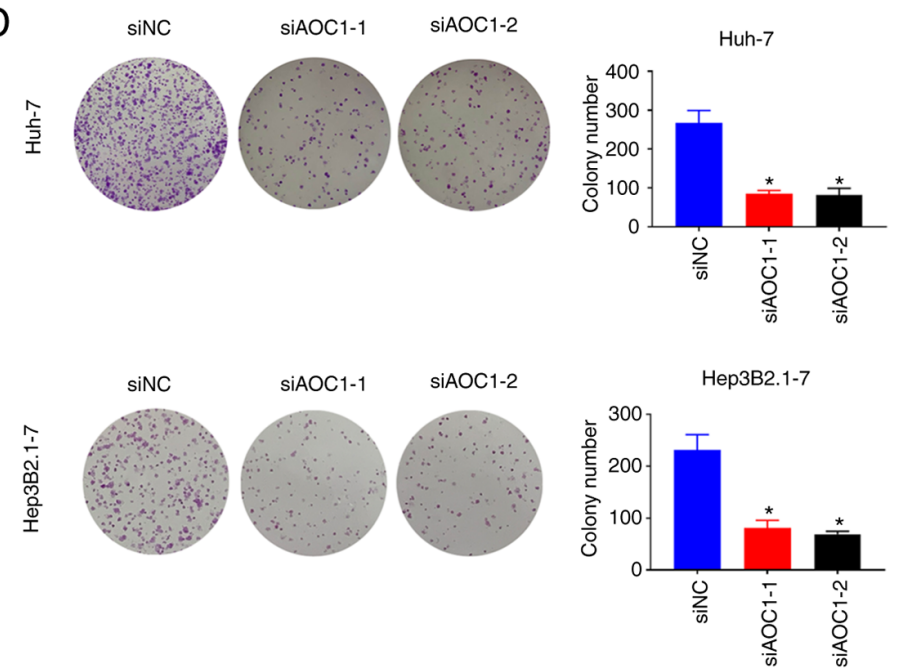

Figure 2. AOC1-knockdown suppresses HCC cell proliferation. (A and B) AOC1-knockdown and overexpression efficiencies were validated by reverse transcription-quantitative PCR and western blotting in Huh-7 and Hep3B2.1-7 cells. (C) Following transfection, cell viability was determined by Cell Counting Kit 8 analysis, and (D) cellular proliferation was measured using a colony formation assay. ${ }^{*} \mathrm{P}<0.05$ vs. the siNC or Vector group. AOC1, amine oxidase copper containing 1; HCC, hepatocellular carcinoma; si, small interfering (RNA); NC, negative control.

As shown in Fig. 3C, E-cadherin expression was increased in Huh-7 and Hep3B2.1-7 cells following AOC1 downregulation, while $\mathrm{N}$-cadherin and vimentin expression was decreased. By contrast, E-cadherin expression was decreased in Huh-7 and Hep3B2.1-7 cells when AOC1 was upregulated, and N-cadherin and vimentin expression were increased. The data indicated that downregulation of $\mathrm{AOC} 1$ expression may decrease the migration and invasiveness of $\mathrm{HCC}$ cells.

AOC1-knockdown inhibits IL-6/JAK/STAT3 pathway activation in HCC cells. The mechanism of AOC1 in HCC was then explored. ROS generation in Huh-7 and Hep3B2.1-7 cells 

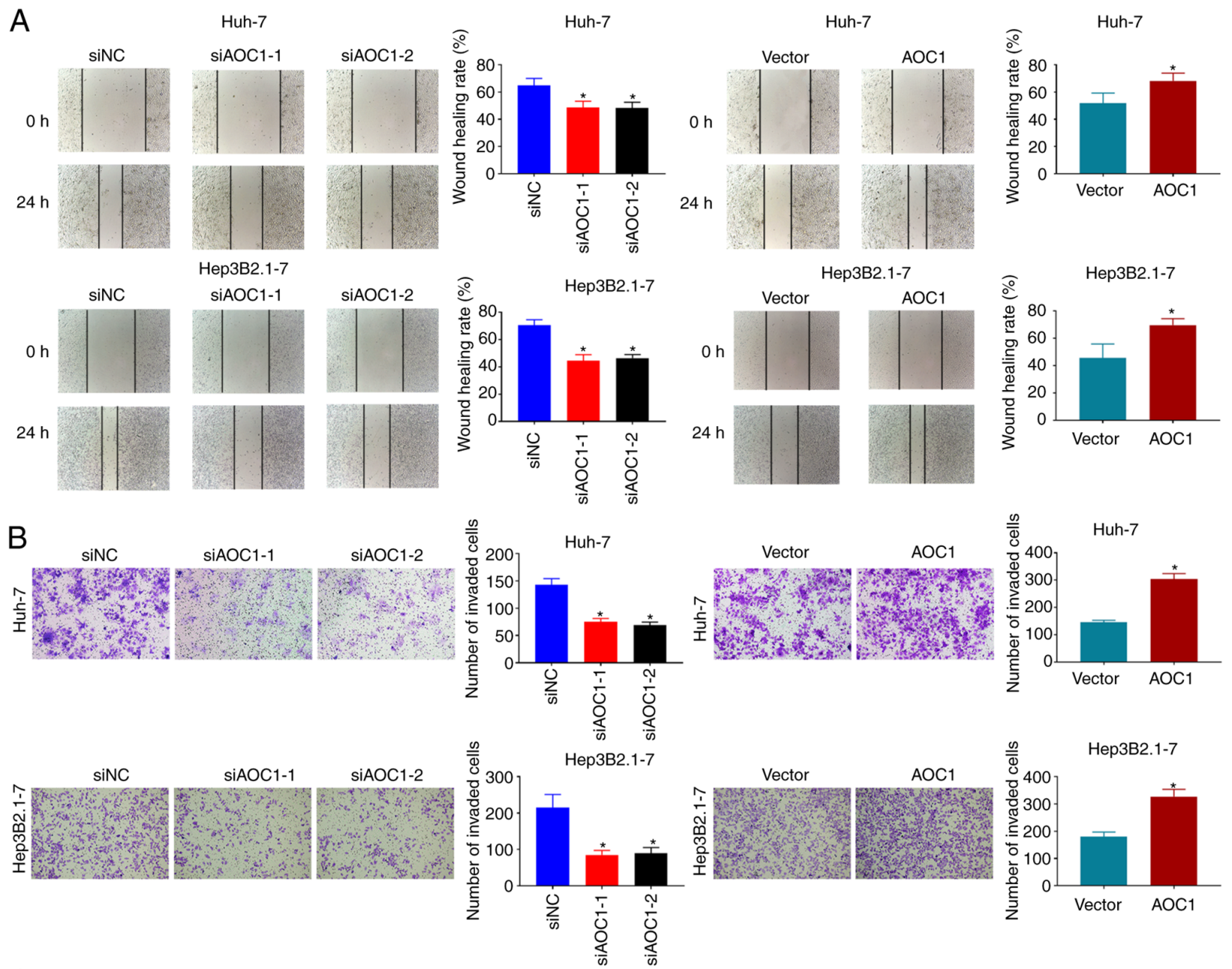

C

Huh-7

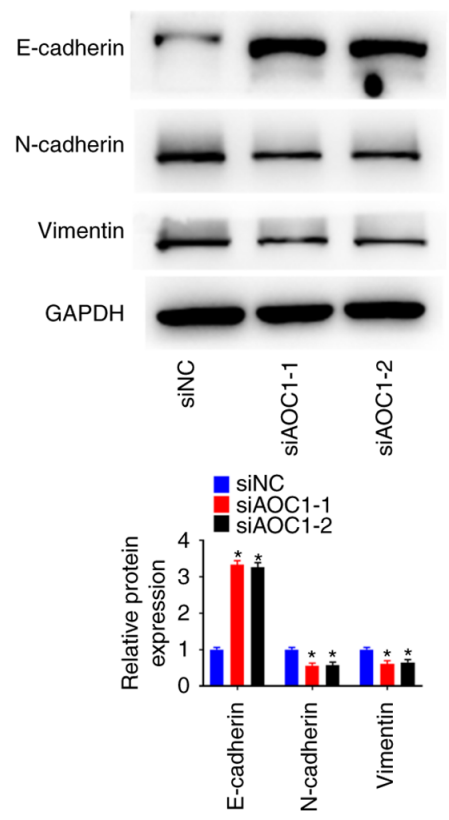

Нер3В2.1-7
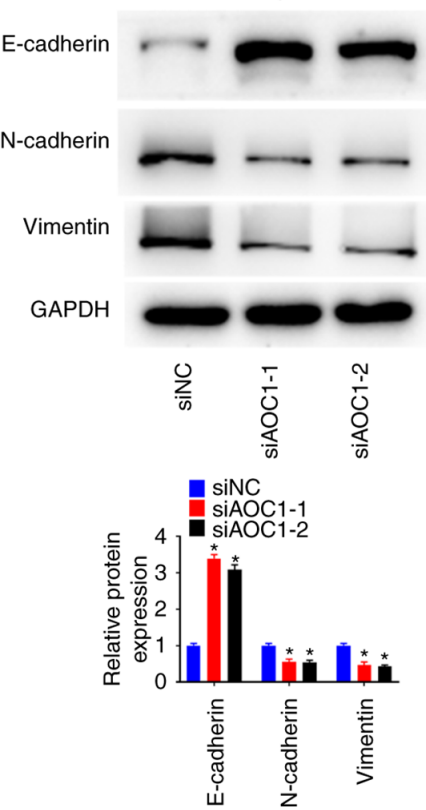

Huh-7

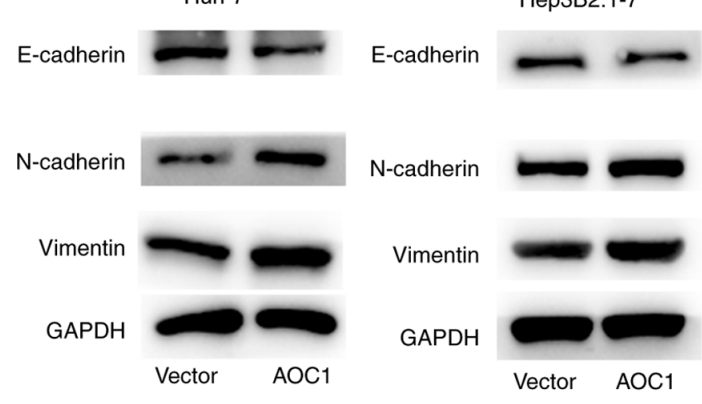

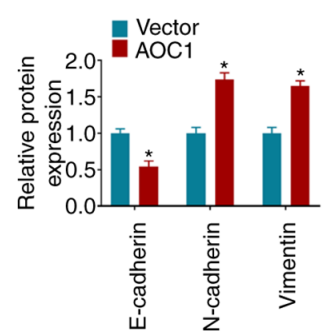

Figure 3. AOC1-knockdown suppresses HCC cell migration and invasiveness. Cellular migration and invasion ability were analyzed using (A) wound-healing and (B) Transwell assays, respectively. (C) Effect of AOC1 on HCC epithelial-mesenchymal transition-related protein levels was determined by western blotting. ${ }^{*} \mathrm{P}<0.05$ vs. the siNC or Vector group. AOC1, amine oxidase copper containing 1; HCC, hepatocellular carcinoma; si, small interfering (RNA); NC, negative control.

was significantly decreased following treatment with both si-AOC1-1 and si-AOC1-2 (Fig. 4A). Furthermore, the GSEA results revealed that the IL-6/JAK/STAT3 pathway was enriched in AOC1-associated HCC (Fig. 4B). Supplementary ELISA results indicated that the amount of IL-6 present in the culture supernatants of HCC cells was reduced by AOC1-knockdown 
A

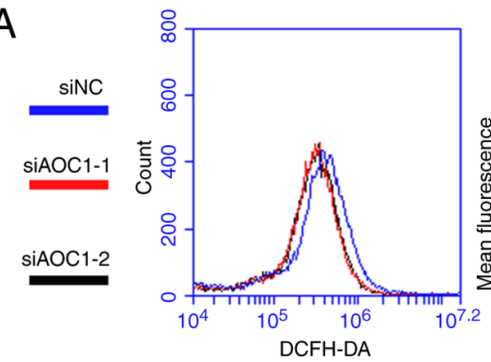

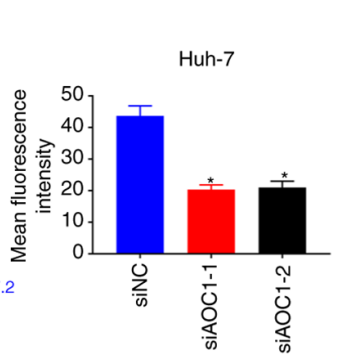
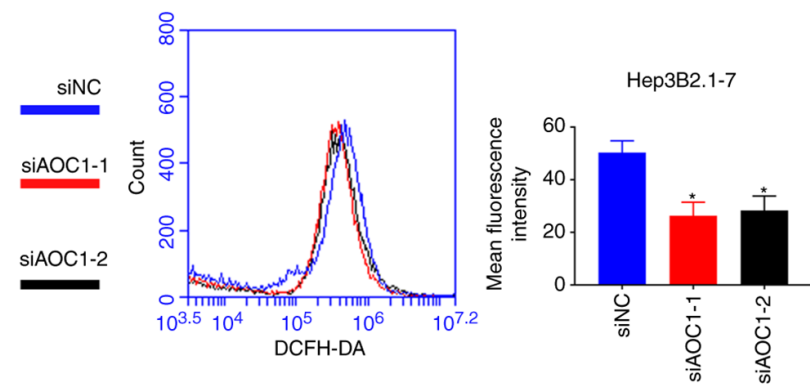

B

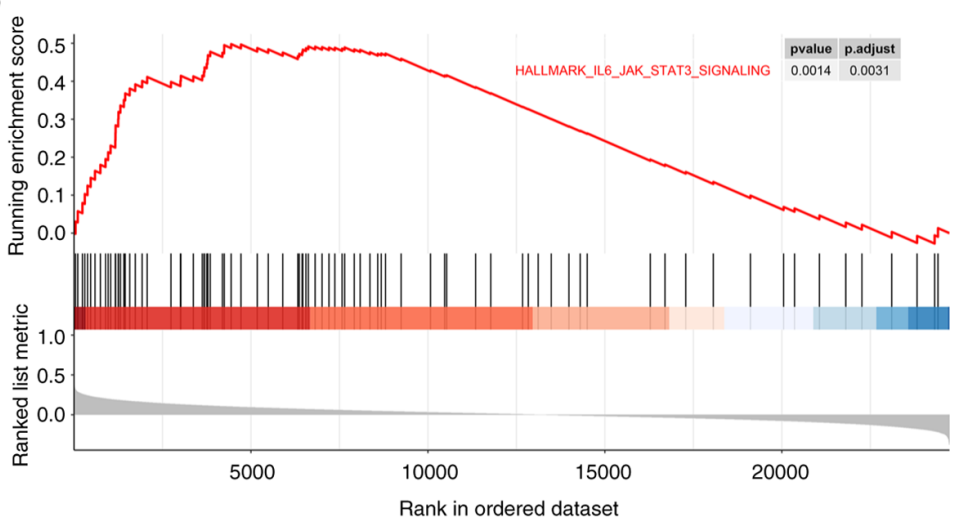

C Huh-7

Нер3В2.1-7
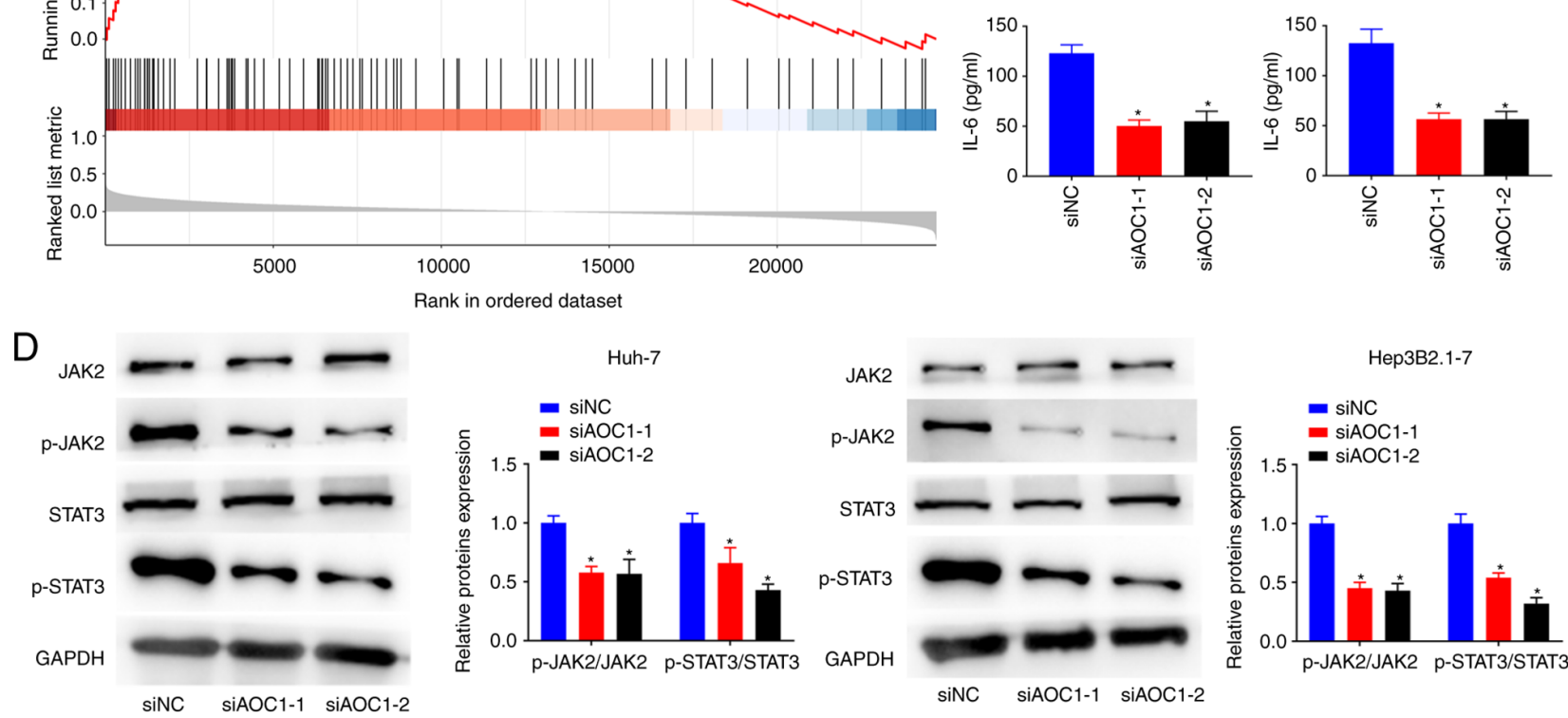

Figure 4. AOC1-knockdown inhibits IL-6/JAK/STAT3 pathway activation in HCC cells. (A) Effect of AOC1-knockdown on HCC cell reactive oxygen species generation. (B) Gene set enrichment analysis results revealed that the IL-6/JAK/STAT3 pathway was enriched in AOC1-related HCC. (C) IL-6 levels in HCC cell lines were analyzed by ELISA. (D) Phosphorylation levels of JAK2 and STAT3 were analyzed by western blotting. * $\mathrm{P}<0.05$ vs. siNC group. AOC1, amine oxidase copper containing 1; HCC, hepatocellular carcinoma; si, small interfering (RNA); NC, negative control.

(Fig. 4C), which also inhibited the phosphorylation of JAK2 and STAT3 (Fig. 4D). The data suggested that AOC1 upregulated the IL-6/JAK/STAT3 pathway in HCC cells.

AOC1-knockdown blocks the IL-6-induced proliferation, migration and invasion abilities of HCC cells. Furthermore, it was investigated whether AOC1 facilitated HCC cell development via the IL-6/JAK/STAT3 pathway. IL-6 treatment promoted the proliferation (Fig. 5A and B), migration (Fig. 5C) and invasion abilities (Fig. 5D) of HCC cells. It was noted that AOCI-knockdown reversed the IL-6-induced proliferation (Fig. 5A and B), migration (Fig. 5C) and invasiveness (Fig. 5D) of HCC Huh-7 and Hep3B2.1-7 cells. The data suggested that AOC1 upregulated the IL-6/JAK/STAT3 pathway to promote HCC cell progression.

\section{Discussion}

AOC1 expression has been reported to be higher in gastric cancer tissues than in normal, noncancer tissues (11). In the present study, AOC1 expression was also found to be upregulated in $\mathrm{HCC}$ tissue samples, and associated with poor clinical outcome in patients with HCC, as well as with tumor differentiation, clinical stage and lymph node metastasis.

There is accumulating evidence to suggest that epithelial-mesenchymal transition (EMT) is involved in HCC metastasis (20). EMT is characterized by the loss of epithelial markers, such as E-cadherin, and the gain of mesenchymal markers, such as vimentin and $\mathrm{N}$-cadherin (21). Previous studies have reported that polyamines are involved in EMT, and in the differentiation of liver epithelial cells (22). Lysyl oxidase-like 2 (LOXL2) is a copper and lysine tyrosyl-quinone-dependent amine oxidase of the LOX family (23), that contributes to the EMT and invasiveness of breast cancer cells (24). Furthermore, AOC1-knockdown was revealed to inhibit EMT in gastric cancer cells (11). The results of the present study also indicated that AOC1 inhibited HCC cell EMT, suggesting that AOC1 promotes the invasiveness of HCC cells.

ROS can function as a double-edged sword in determining the fate of tumor cells, exhibiting both pro-and anti-tumorigenic effects (25). In the current study, ROS production in $\mathrm{HCC}$ cells was reduced by AOC1 downregulation, thus 
A

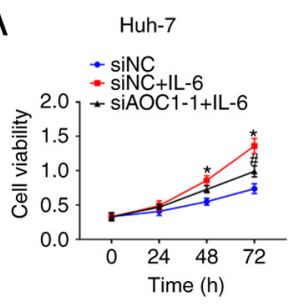

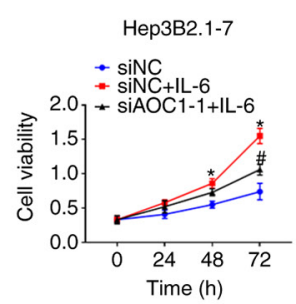

B

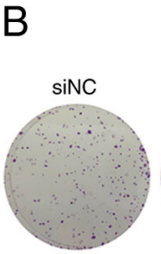

Huh-7

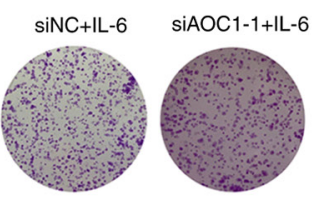

C

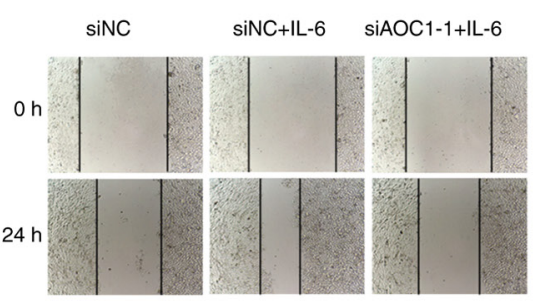

Huh-7
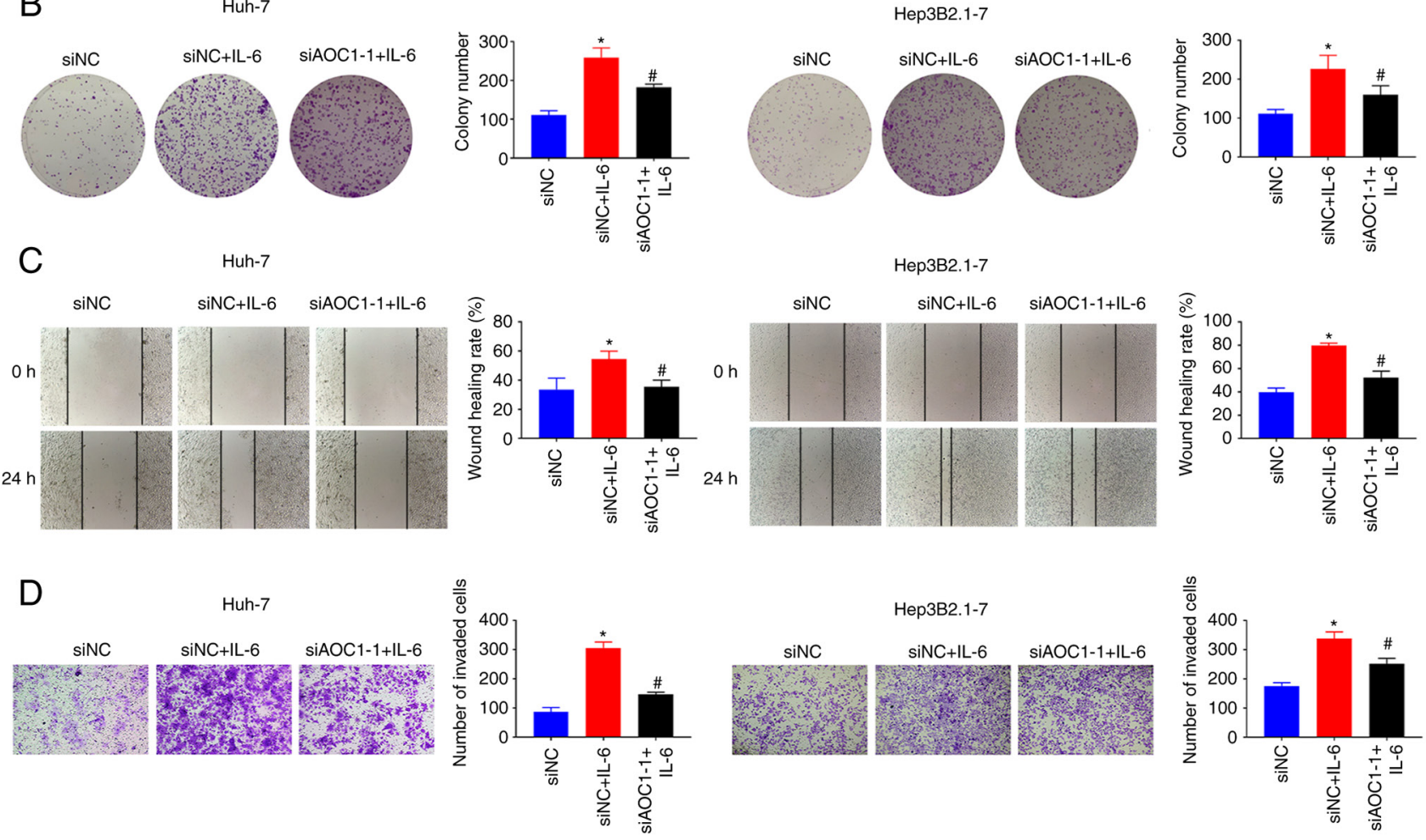

Figure 5. AOC1-knockdown blocks the IL-6-induced proliferation, migration and invasiveness in HCC cell lines. HCC cell (A) viability, (B) proliferation, (C) migration and (D) invasiveness were analyzed following IL-6 treatment using Cell Counting Kit 8, colony formation, wound-healing and Transwell assays, respectively. ${ }^{*} \mathrm{P}<0.05$ vs. siNC group; ${ }^{\#} \mathrm{P}<0.05$ vs. siNC + IL-6 group. AOC1, amine oxidase copper containing 1 ; HCC, hepatocellular carcinoma; si, small interfering (RNA); NC, negative control.

inhibiting IL-6 expression. Consistent with these results, the disintegrin MMP9 and NADPH oxidase coordinate to induce ROS generation and promote IL-6-induced EMT by activating the JNK signaling pathway (26). Furthermore, TGF- $\beta 1$ enhanced pancreatic cancer cell invasiveness by generating ROS through NADPH oxidase, resulting in NF-kB activation, IL-6 generation and an increase in the expression of MMP2 (27).

IL-6 also induces cellular oxidative stress, promoting cancer progression (28). IL-6 is a key immunoregulatory cytokine in the tumor microenvironment, and has been regarded as a trigger of EMT, stimulating tumor growth and metastasis (29). IL-6-induced activation of the JAK/STAT pathway, particularly STAT3, plays a vital role in tumorigenic processes (30). As such, IL-6-induced EMT, cellular migration and invasiveness were inhibited by the inactivation of the JAK2/STAT3 pathway in HCC and pancreatic cancer $(31,32)$ The results of the present study indicated that decreased $\mathrm{AOC1}$ expression inactivated the JAK/STAT signaling pathway. Moreover, AOC1-knockdown reversed the HCC cell proliferation, invasion and migration properties induced by IL-6, suggesting that $\mathrm{AOC1}$ plays a role in $\mathrm{HCC}$ progression and prognosis.
In conclusion, the results of the current study demonstrate that $\mathrm{AOC} 1$ promoted the proliferation, migration and invasiveness of HCC cells by regulating the IL-6/JAK/STAT3 pathway. Limitations of the present study included the sole use of in vitro cell experiments. Therefore, in vivo experiments are required to assess the role of the $\mathrm{AOC} 1$ in tumor progression, as well as the underlying mechanism. AOC1 may be a potential therapeutic and prognostic target for HCC, and its clinical value should be further investigated.

\section{Acknowledgements}

Not applicable.

\section{Funding}

No funding was received.

\section{Availability of data and material}

The datasets used and/or analyzed during the current study are available from the corresponding author on reasonable request. 


\section{Authors' contributions}

QD and CJ designed the study. DL, YZ and FL performed the experiments. JL, JD and JC analyzed the data. QD and DL confirm the authenticity of all the raw data. JC and CJ wrote the paper. QD and DL revised the paper. All authors approved the final version of the manuscript.

\section{Ethics approval and consent to participate}

The present study was authorized by the ethics committee of Qingdao No. 6 People's Hospital, and all patients provided written informed consent.

\section{Patient consent for publication}

Not applicable.

\section{Competing interests}

The authors declare that they have no competing interests.

\section{References}

1. Bray F, Ferlay J, Soerjomataram I, Siegel RL, Torre LA and Jemal A: Global cancer statistics 2018: GLOBOCAN estimates of incidence and mortality worldwide for 36 cancers in 185 countries. CA Cancer J Clin 68: 394-424, 2018.

2. Harris PS, Hansen RM, Gray ME, Massoud OI, McGuire BM and Shoreibah MG: Hepatocellular carcinoma surveillance: An evidence-based approach. World J Gastroenterol 25: 1550-1559, 2019.

3. Petruzziello A: Epidemiology of hepatitis B virus (HBV) and hepatitis C Virus (HCV) related hepatocellular carcinoma. Open Virol J 12: 26-32, 2018.

4. Liu CY, Chen KF and Chen PJ: Treatment of liver cancer. Cold Spring Harb Perspect Med 5: a021535, 2015.

5. Wang EA, Stein JP, Bellavia RJ and Broadwell SR: Treatment options for unresectable HCC with a focus on SIRT with Yttrium-90 resin microspheres. Int J Clin Pract 71: 30, 2017.

6. Hartke J, Johnson M and Ghabril M: The diagnosis and treatment of hepatocellular carcinoma. Semin Diagn Pathol 34: 153-159, 2017.

7. Toninello A, Pietrangeli $\mathrm{P}$, De Marchi U, Salvi M and Mondovì $\mathrm{B}$ Amine oxidases in apoptosis and cancer. Biochim Biophys Acta 1: 1-13, 2006.

8. Schwelberger HG: Structural organization of mammalian copper-containing amine oxidase genes. Inflamm Res 59 (Suppl 2): S223-S235, 2010.

9. Sun WY, Choi J, Cha YJ and Koo JS: Evaluation of the expression of amine oxidase proteins in breast cancer. Int J Mol Sci 18: $2775,2017$.

10. Vakal S, Jalkanen S, Dahlström KM and Salminen TA: Human copper-containing amine oxidases in drug design and development. Molecules 25: 1293, 2020.

11. Xu F, Xu Y, Xiong JH, Zhang JH, Wu J, Luo J and Xiong JP: AOC1 contributes to tumor progression by promoting the AKT and EMT pathways in gastric cancer. Cancer Manag Res 12: 1789-1798, 2020.

12. Nourbakhsh M, Farzaneh S, Taghikhani A, Zarghi A and Noori S: The effect of a newly synthesized ferrocene derivative against MCF-7 breast cancer cells and spheroid stem cells through ROS production and inhibition of JAK2/STAT3 signaling pathway. Anticancer Agents Med Chem 20: 875-886, 2020

13. Cao Y, Wang J, Tian H and Fu GH: Mitochondrial ROS accumulation inhibiting JAK2/STAT3 pathway is a critical modulator of CYT997-induced autophagy and apoptosis in gastric cancer. J Exp Clin Cancer Res 39: 119, 2020.
14. Li J, Pu T, Yin L, Li Q, Liao CP and Wu BJ: MAOA-mediated reprogramming of stromal fibroblasts promotes prostate tumorigenesis and cancer stemness. Oncogene 39: 3305-3321, 2020.

15. Lee HM, Sia APE, Li L, Sathasivam HP, Chan MSA, Rajadurai P, Tsang CM, Tsao SW, Murray PG, Tao Q, et al: Monoamine oxidase A is down-regulated in EBV-associated nasopharyngeal carcinoma. Sci Rep 10: 6115, 2020

16. Therneau TM: Survival Analysis [R package survival version 2.41-3]. Technometrics 46: 111-112, 2015.

17. Li ZS and Li Q: The latest 2010 WHO classification of tumors of digestive system. Zhonghua Bing Li Xue Za Zhi 40: 351-354, 2011 (In Chinese).

18. Hoshi T, Watanabe Miyano S, Watanabe H, Sonobe RMK, Seki Y, Ohta E, Nomoto K, Matsui J and Funahashi Y: Lenvatinib induces death of human hepatocellular carcinoma cells harboring an activated FGF signaling pathway through inhibition of FGFR-MAPK cascades. Biochem Biophys Res Commun 513: 1-7, 2019.

19. Livak KJ and Schmittgen TD: Analysis of relative gene expression data using real-time quantitative PCR and the 2(-Delta Delta C(T)) method. Methods 25: 402-408, 2001.

20. Jiang H, Zhou Z, Jin S, Xu K, Zhang H, Xu J, Sun Q, Wang J and Xu J: PRMT9 promotes hepatocellular carcinoma invasion and metastasis via activating PI3K/Akt/GSK-3 $\beta /$ Snail signaling. Cancer Sci 109: 1414-1427, 2018.

21. Giannelli G, Koudelkova P, Dituri F and Mikulits W: Role of epithelial to mesenchymal transition in hepatocellular carcinoma. J Hepatol 65: 798-808, 2016.

22. Ivanova ON, Snezhkina AV, Krasnov GS, Valuev-Elliston VT, Khomich OA, Khomutov AR and Keinanen TA: Activation of

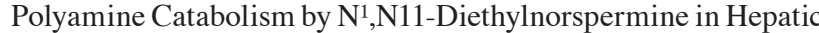
HepaRG cells induces dedifferentiation and mesenchymal-like phenotype. Cells 7: 275, 2018.

23. Wen B, Xu LY and Li EM: LOXL2 in cancer: Regulation, downstream effectors and novel roles. Biochim Biophys Acta Rev Cancer 1874: 188435, 2020.

24. Moon HJ, Finney J, Xu L, Moore D, Welch DR and Mure M: MCF-7 cells expressing nuclear associated lysyl oxidase-like 2 (LOXL2) exhibit an epithelial-to-mesenchymal transition (EMT) phenotype and are highly invasive in vitro. J Biol Chem 288: 30000-30008, 2013.

25. Moloney JN and Cotter TG: ROS signalling in the biology of cancer. Semin Cell Dev Biol 80: 50-64, 2018

26. Dong Y, Wu Z, He M, Chen Y, Chen Y, Shen X, Zhao X, Zhang L, Yuan B and Zeng Z: ADAM9 mediates the interleukin-6-induced Epithelial-Mesenchymal transition and metastasis through ROS production in hepatoma cells. Cancer Lett 421: 1-14, 2018.

27. Binker MG, Binker-Cosen AA, Gaisano HY, de Cosen RH and Cosen-Binker LI: TGF- $\beta 1$ increases invasiveness of SW1990 cells through Rac1/ROS/NF-kB/IL-6/MMP-2. Biochem Biophys Res Commun 405: 140-145, 2011.

28. Zhang Y, Yan W, Collins MA, Bednar F, Rakshit S, Zetter BR, Stanger BZ, Chung I, Rhim AD and di Magliano MP: Interleukin- 6 is required for pancreatic cancer progression by promoting MAPK signaling activation and oxidative stress resistance. Cancer Res 73: 6359-6374, 2013.

29. Browning L, Patel MR, Horvath EB, Tawara K and Jorcyk CL: IL-6 and ovarian cancer: Inflammatory cytokines in promotion of metastasis. Cancer Manag Res 10: 6685-6693, 2018.

30. Bromberg J and Wang TC: Inflammation and cancer: IL-6 and STAT3 complete the link. Cancer Cell 15: 79-80, 2009.

31. Gao Y, Li W, Liu R, Guo Q, Li J, Bao Y, Zheng H, Jiang S and Hua B: Norcantharidin inhibits IL-6-induced epithelial-mesenchymal transition via the JAK2/STAT3/TWIST signaling pathway in hepatocellular carcinoma cells. Oncol Rep 38: 1224-1232, 2017.

32. Chen J, Wang S, Su J, Chu G, You H, Chen Z, Sun H, Chen B and Zhou M: Interleukin-32 $\alpha$ inactivates JAK2/STAT3 signaling and reverses interleukin-6-induced epithelial-mesenchymal transition, invasion, and metastasis in pancreatic cancer cells. Onco Targets Ther 9: 4225-4237, 2016.

This work is licensed under a Creative Commons Attribution-NonCommercial-NoDerivatives 4.0 International (CC BY-NC-ND 4.0) License. 